\title{
Numerical analysis of forced convection heat transfer in a rectangular micro-channel totally filled with Ag/ water nano fluid in slip flow regime using the lattice Boltzmann method
}

Kaouther Ben Ltaifa ${ }^{1^{*}}$, Annunziata D'Orazio ${ }^{2}$, Arash Karimipour ${ }^{3}$, and Hacen Dhahri ${ }^{1}$

${ }^{1}$ University of Monastir, National Engineering School of Monastir, Street Ibn Eljazza, 5019 Monastir, Tunisia.

${ }^{2}$ Dipartimento di Ingegneria Astronautica, Elettrica ed Energetica, Sapienza Universita di Roma, Via Eudossiana 18, Roma 00184, Italy.

${ }^{3}$ Department of Mechanical Engineering, Najafabad Branch, Islamic Azad University, Najafabad, Iran.

\begin{abstract}
Numerical simulation reported on heat transfer and fluid flow in a two-dimensional rectangular micro channel totally filled with $\mathrm{Ag} /$ water. The first - order slip/jump boundary conditions were uniformly imposed to the up and bottom walls. The governing conservation equations are translated in dimensionless form using the thermal Single Relaxation Time (T-SRT) modified Lattice Boltzmann Method (LBM) with double distribution functions (DDFs). The viscous dissipations effects are adopted into the energy equation. Effects of nanoparticle volume fraction $\varphi$, slip coefficient, B, on the flow of Nano fluid and heat transfer were studied. The results were interpreter in terms of slip velocity; temperature jump and Nusselt number. Based on the results found, it can be concluded that decreasing the values of slip coefficient enhances the convective heat transfer coefficient and consequently the Nusselt number $(\mathrm{Nu})$ but increases the slip velocity at the wall and temperature jump values.
\end{abstract}

\footnotetext{
* Corresponding author: Kaouther.benltaifa@yahoo.com
} 


\section{Nomenclature}

\begin{tabular}{|c|c|}
\hline$B=\beta / h$ & Dimensionless slip coefficient \\
\hline$D_{H}=2 h$ & Hydraulic diameter $(m)$ \\
\hline$c$ & Lattice speed $\left(m . s^{-1}\right)$ \\
\hline$d_{f}$ & $\begin{array}{l}\text { Molecular diameter of the base } \\
\text { (pure water) },(\mathrm{nm})\end{array}$ \\
\hline$d_{p}$ & Nanoparticle diameter $(\mathrm{nm})$ \\
\hline$e$ & density of internal energy \\
\hline$c_{i}$ & Discrete velocity in direction $i$ \\
\hline$f_{i}, g_{i}$ & Distribution function in direction $i$ \\
\hline$f_{i}^{e q}, g_{i}^{e q}$ & Equilibrium distribution function in direction $i$ \\
\hline$H, L$ & $\begin{array}{l}\text { Dimensionless height and length of the } \\
\text { microchannel }(\mathrm{m})\end{array}$ \\
\hline$f_{i}, g_{i}$ & Modified distribution functions \\
\hline$L_{s}$ & Slip length $(m)$ \\
\hline$Z_{i}$ & Heat dissipation \\
\hline $\mathrm{Nu}_{\mathrm{x}}$ & $\begin{array}{l}\text { Local Nusselt number along the microchannel } \\
\text { wall }\end{array}$ \\
\hline$R$ & Gas constant \\
\hline $\operatorname{Pr}$ & Prandtl number, $\operatorname{Pr}=v_{\mathrm{nf}} / \alpha_{n f}$ \\
\hline $\operatorname{Re}$ & Reynolds number, $\operatorname{Re}=\rho_{\mathrm{nf}} u_{n f} D_{H} / \mu_{n f}$ \\
\hline$T_{i}$ & Inlet temperature $(K)$ \\
\hline$T_{w}$ & Wall temperature $(K)$ \\
\hline$r$ & Accommodation coefficient \\
\hline$t$ & Time (s) \\
\hline$u, v$ & Velocity $\left(m \cdot s^{-1}\right)$ \\
\hline$U, V$ & Dimensionless velocity \\
\hline$x, y$ & Cartesian coordinates $(m)$ \\
\hline$X, Y$ & Dimensionless coordinates \\
\hline$u_{i}$ & Inlet flow velocity $\left(m \cdot s^{-1}\right)$ \\
\hline$U_{s}$ & Dimensionless slip velocity \\
\hline \multicolumn{2}{|l|}{ Greek symbols } \\
\hline$\theta=T / T_{i}$ & Dimensionless temperature \\
\hline$\Delta x$ & Lattice step \\
\hline
\end{tabular}




\begin{tabular}{|cl|}
$\Delta t$ & Time step \\
$K$ & Thermal conductivity $\left(W \cdot m^{-1} \cdot K^{-1}\right)$ \\
$\varphi$ & Volume fraction of Argent \\
$\alpha$ & Thermal diffusivity $\left(m^{2} \cdot s^{-1}\right)$ \\
$\beta$ & Sip coefficient \\
$\mu$ & Dynamic viscosity $\left(K g \cdot m^{-1} \cdot s^{-1}\right)$ \\
$v$ & Kinematic viscosity $\left(m^{2} \cdot s^{-1}\right)$ \\
$\rho$ & Density $\left(K g \cdot m^{-3}\right)$ \\
$\zeta$ & Temperature jump distance \\
$\Omega$ & Collision operator \\
$\tau_{f}, \tau_{g}$ & Relaxation times for momentum and internal \\
$w_{i}$ & energy \\
Subscripts & Weight coefficient in direction $i$ \\
$\mathrm{f}$ & \\
$\mathrm{nf}$ & Base fluid \\
$\mathrm{e}$ & Nanofluid \\
$\mathrm{s}$ & Equilibrium \\
$\mathrm{w}$ & Solid \\
$\mathrm{i}$ & Wall \\
out & Inlet \\
\hline
\end{tabular}

\section{Introduction}

Heat transfer in fluids led to many practical and industrial applications, including transportation (combustion engines), energy supply, air conditioning, and electronics cooling... The recent considerable development of research treating with nanofluid for certain applications induce an improvement of heat transfers by introducing into a pure 
fluid a low concentration of nanoparticles. The intensity of heat transfer depends strongly on the conductivity and thermal capacity of the heat transfer fluids. Then, nanofluids are colloidal solutions obtained by dispersing solid particles of nanometric size in a base fluid. Therefore, some of these solutions have been found to contribute more in the enhancement of heat transfer under certain conditions.

There are many studies concerning nanofluid in different geometries, some researchers have reported the flow and heat transfer of the nanofluid in microchannels [1-4]. For instance, Karimipour et al.[5] simulated the $\mathrm{Cu}$-water nanofluid in a microchannel for slip condition. In addition, the lattice Boltzmann method are sued to simulate each problems related to heat transfer of nanofluids in microchannels $[6,7]$.

According to the above literature, the present study deals with laminar forced convection heat transfer of Ag-Water nanofluids in a microchannel using Lattice Boltzmann method. Our attention focused on the effects of emerging parameter's on the slip velocity, temperature jump and Nusselt number.

\section{Problem statement}

\subsection{Geometry details}

The schematic diagram of the present computational domain is illustrated in Figure 2. The velocity and temperature profiles at the inlet are considered as $u_{i}$ and $T_{i}$. The wall temperature is set to $T_{w}=2 T_{i}$. The open straight channel of height $\mathrm{H}$ consists of upper and lower walls of length L.In the present simulation Reynolds number is fixed to be 0,01 .

\section{2 . Lattice Boltzmann model}

The Lattice Boltzmann equation with a single relaxation time from the BGK model was adopted in this study and it can be expressed as:

$$
\begin{aligned}
& \tilde{f}_{i}\left(\vec{x}+\vec{c}_{i} d t, t+d t\right)-\tilde{f}_{i}(\vec{x}, t)=-\frac{d t}{\tau_{f}+0.5 d t}\left(\tilde{f}_{i}-f_{i}^{e}\right) \\
& \tilde{g}_{i}\left(\vec{x}+\vec{c}_{i} d t, t+d t\right)-\tilde{g}_{i}(\vec{x}, t)=-\frac{d t}{\tau_{g}+0.5 d t}\left(\tilde{g}_{i}-g_{i}^{e}\right)-\frac{\tau_{g} d t}{\tau_{g}+0.5 d t} f_{i} Z_{i}
\end{aligned}
$$

$\mathrm{f}$ and $\mathrm{g}$ indicated the density momentum and internal energy distribution functions respectively.The discrete distribution function $\tilde{f}_{i}$ and $\tilde{g}_{i}$ with velocity $c_{i}$ at position $x$ and time $\mathrm{t}$ are given by [8]:

$$
\begin{aligned}
& \tilde{f}_{i}=f_{i}+\frac{d t}{2 \tau_{f}}\left(f_{i}-f_{i}^{e}\right) \\
& \tilde{g}_{i}=g_{i}+\frac{d t}{2 \tau_{g}}\left(g_{i}-g_{i}^{e}\right)+\frac{d t}{2} f_{i} Z_{i}
\end{aligned}
$$


$Z_{i}=\left(\vec{c}_{i}-\vec{u}\right) \cdot D_{i} \vec{u}, D_{i}=\partial_{t}+\vec{c}_{i} \cdot \nabla$

$Z_{i}$ and $D_{i}$ represent the effect of heat dissipation and the material derivative along the direction $\overrightarrow{c_{i}}, \tau_{g}$ and $\tau_{g}$ are the hydrodynamic and thermal relaxation times, respectively. $f^{e}{ }_{i}$ and $g^{e}{ }_{i}$ are the equilibrium distribution function.

In this study, the 2-D nine-bit model (D2Q9) is used (Fig.1).

This model can be defined as :

$$
\begin{aligned}
& f_{i}^{e}=\omega_{i} \rho\left[1+\frac{3 \vec{c}_{i} \cdot \vec{u}}{c^{2}}+\frac{9\left(\vec{c}_{i} \cdot \vec{u}\right)^{2}}{2 c^{4}}-\frac{3\left(u^{2}+v^{2}\right)}{2 c^{2}}\right] \\
& g_{0}^{e}=-\omega_{0}\left[\frac{3 \rho e\left(u^{2}+v^{2}\right)}{2 c^{2}}\right] \\
& g_{0}^{e} g_{1,2,3,4}^{e}=\omega_{i} \rho\left[1.5+1.5 \frac{\vec{c}_{i} \cdot \vec{u}}{c^{2}}+4.5 \frac{\left(\vec{c}_{i} \cdot \vec{u}\right)^{2}}{c^{4}}-1.5 \frac{\left(u^{2}+v^{2}\right)}{c^{2}}\right] \\
& g_{5,6,7,8}^{e}=\omega_{i} \rho\left[3+6 \frac{\vec{c}_{i} \cdot \vec{u}}{c^{2}}+4.5 \frac{\left(\vec{c}_{i} \cdot \vec{u}\right)^{2}}{c^{4}}-1.5 \frac{\left(u^{2}+v^{2}\right)}{c^{2}}\right]
\end{aligned}
$$

where

$$
\vec{u}=(u, v), \vec{x}=(x, y), \rho e=\rho R T, c^{2}=3 R T \text { and } \mathrm{T} \text { is the temperature, } w_{i} \text { are set as : }
$$
$w_{0}=4 / 9, w_{1,4}=1 / 9$ and $w_{5,8}=1 / 36$. The D2Q9-discret velocity model is given as [9]:

$$
\begin{aligned}
& \vec{c}_{o}=(0,0) \\
& \vec{c}_{i}=\left(\cos \frac{i-1}{2} \pi, \sin \frac{i-1}{2} \pi\right) c, i=1,2,3,4 \\
& \vec{c}_{i}=\sqrt{2}\left(\cos \left(\frac{i-5}{2} \pi+\frac{\pi}{4}\right), \sin \left(\frac{i-5}{2} \pi+\frac{\pi}{4}\right)\right) c, i=5,6,7,8
\end{aligned}
$$

The hydrodynamic and thermal variables can be determined by [10]:

$$
\rho=\sum_{i} \tilde{f}_{i}
$$

$$
\rho \vec{u}=\sum_{i} \vec{c}_{i} \tilde{f}_{i}
$$


$\rho e=\sum_{i} \tilde{g}_{i}-\frac{d t}{2} \sum_{i} f_{i} Z_{i}$

The kinematic viscosity and the thermal diffusivity are given by :

$v=\tau_{f} R \bar{T}, \quad \alpha=2 \tau_{g} R \bar{T}$

\section{Nanofluid}

The properties of the nanofuid are obtained using the following relations [11] :

$\rho_{n f}=\varphi \rho_{s}+(1-\varphi)_{\rho_{f}}$

$\left(\rho c_{p}\right)_{n f}=\varphi\left(\rho c_{p}\right)_{s}+(1-\varphi)\left(\rho c_{p}\right)_{f}$

$\alpha_{n f}=k_{n f} /\left(\rho c_{p}\right)_{n f}$

Using the Brinkman model [11] the effettive dynamic viscosity is:

$\mu_{n f}=\mu_{f} /(1-\varphi)^{2.5}$

The nanofluid thermal conductivity as a function of liquid and solid conductivities is expressed as following [11]:

$$
k_{n f}=k_{f}\left(1+\frac{k_{s} A_{s}}{k_{f} A_{f}} c k_{s} P_{e} k_{s} \frac{A_{s}}{k_{f} k_{f}}\right)
$$

Where

$\frac{A_{s}}{A_{f}}=\frac{d_{f}}{d_{s}} \frac{\varphi}{1-\varphi} \quad P_{e}=\frac{u_{B} d_{s}}{\alpha_{f}} \quad u_{B}=\frac{2 k_{B} T}{\pi \mu_{f} d_{s}{ }^{2}}$

$k_{B}=1.3807 \times 10^{-23} \mathrm{~J} / \mathrm{K}$

$$
C=36000
$$

In wich $P_{e}$ is the Peclet number with the brownien motion velocity of particles $u_{B}$ and $k_{B}$ is the constant of Boltzmann.

\subsection{Boundary condition}

Non-equilibrium bounce back model, normal to the boundary, is used for inlet and outlet hydrodynamic boundary conditions. In this model, distribution functions are reflected in suitable ways to satisfy the equilibrium conditions and improve accuracy. 


$$
\begin{aligned}
& \tilde{f}_{1}=\tilde{f}_{3}+\frac{2}{3} \rho_{\text {in }} u_{\text {in }} \\
& \tilde{f}_{5}=\tilde{f}_{7}+\frac{1}{2}\left(\tilde{f}_{4}-\tilde{f}_{2}\right)+\frac{1}{6} \rho_{\text {in }} u_{\text {in }} \\
& \tilde{f}_{8}=\tilde{f}_{6}-\frac{1}{2}\left(\tilde{f}_{4}-\tilde{f}_{2}\right)+\frac{1}{6} \rho_{\text {in }} u_{\text {in }} \\
& \tilde{f}_{3}=\tilde{f}_{1}-\frac{2}{3} \rho_{\text {out }} u_{\text {out }} \\
& \tilde{f}_{7}=\tilde{f}_{5}-\frac{1}{2}\left(\tilde{f}_{4}-\tilde{f}_{2}\right)-\frac{1}{6} \rho_{\text {out }} u_{\text {out }}-\frac{1}{2} \rho_{\text {out }} u_{\text {out }} \\
& \tilde{f}_{6}=\tilde{f}_{8}+\frac{1}{2}\left(\tilde{f}_{4}-\tilde{f}_{2}\right)-\frac{1}{6} \rho_{\text {out }} u_{\text {out }}+\frac{1}{2} \rho_{\text {out }} u_{\text {out }}
\end{aligned}
$$

The unknown inlet and outlet thermal distribution functions are estimated using the known inlet temperature profile and nnon-equilibrium bounce back model as follow [12,13]:

$$
\begin{aligned}
& \tilde{g}_{1}=\frac{6 \rho_{i n} e_{i n}+3 d t \sum_{i}\left[f_{i} Z_{i}-6\left(g_{0}+\tilde{g}_{2}+\tilde{g}_{3}+\tilde{g}_{4}+\tilde{g}_{6}+\tilde{g}_{7}\right)\right]}{2+3 u_{i n}+3 u_{i n}^{2}} \times \\
& {\left[1.5+1.5 u_{\text {in }}+3 u_{\text {in }}^{2}\right] \frac{1}{9}} \\
& \tilde{g}_{5}=\frac{6 \rho_{i n} e_{i n}+3 d t \sum_{i}\left[f_{i} Z_{i}-6\left(\tilde{g}_{0}+\tilde{g}_{2}+\tilde{g}_{3}+\tilde{g}_{4}+\tilde{g}_{6}+\tilde{g}_{7}\right)\right]}{2+3 u_{i n}+3 u_{i n}^{2}} \times \\
& {\left[3+6 u_{i n}+3 u_{i n}^{2}\right] \frac{1}{36}} \\
& \tilde{g}_{8}=\frac{6 \rho_{i n} e_{i n}+3 d t \sum_{i}\left[f_{i} Z_{i}-6\left(\tilde{g}_{0}+\tilde{g}_{2}+\tilde{g}_{3}+\tilde{g}_{4}+\tilde{g}_{6}+\tilde{g}_{7}\right)\right]}{2+3 u_{i n}+3 u_{i n}^{2}} \times \\
& {\left[3+6 u_{i n}+3 u_{i n}^{2}\right] \frac{1}{36}} \\
& \tilde{g}_{6}=\frac{6\left(\tilde{g}_{1}+\tilde{g}_{5}+\tilde{g}_{8}\right)-3 d t \sum_{i}\left(\frac{c_{i x}}{c}\right) f_{i} Z i-6\left(\rho e u_{\text {out }}\right)}{2-3 u_{\text {out }}+3 u_{\text {out }}^{2}} \times \\
& {\left[3-6 u_{\text {out }}+6 v_{\text {out }}+3\left(u_{\text {out }}^{2}+v_{\text {out }}^{2}\right)-9 u_{\text {out }} v_{\text {out }}\right] \frac{1}{36}} \\
& \tilde{g}_{3}=\frac{6\left(\tilde{g}_{1}+g_{5}+\tilde{g}_{8}\right)-3 d t \sum_{i}\left(\frac{c_{i x}}{c}\right) f_{i} Z i-6\left(\rho e u_{\text {out }}\right)}{2-3 u_{\text {out }}+3 u_{\text {out }}^{2}} \times \\
& {\left[1.5-1.5 u_{\text {out }}+3 u_{\text {out }}^{2}-1.5 v_{\text {out }}^{2}\right] \frac{1}{9}} \\
& \tilde{g}_{7}=\frac{6\left(\tilde{g}_{1}+g_{5}+\tilde{g}_{8}\right)-3 d t \sum_{i}\left(\frac{c_{i x}}{c}\right) f_{i} Z i-6\left(\rho e u_{\text {out }}\right)}{2-3 u_{\text {out }}+3 u_{\text {out }}^{2}} \times \\
& {\left[3-6 u_{\text {out }}-6 v_{\text {out }}+3\left(u_{\text {out }}^{2}+v_{\text {out }}^{2}\right)+9 u_{\text {out }} v_{\text {out }}\right] \frac{1}{36}}
\end{aligned}
$$


Concerning the boundary conditions of the walls of the microchannels, the boundary condition of sliding is applied for the hydrodynamic field. Ngoma and Erchiqui [14] considered $\beta$ for the slip length coefficient and defined the slip velocity $u_{s}$ for the liquid inside the microchannel on the fixed walls as follows :

$u_{s}= \pm\left.\beta \frac{\partial u_{\text {fluid }}}{\partial y}\right|_{y=0, h}$

The dimensionless form is written as :

$$
U_{s}= \pm\left. B \frac{\partial U}{\partial Y}\right|_{\mathrm{Y}=0,1}
$$

To defined the slip velocity in LBM, the specular reflective bounce back model (combination of bounce back and specular boundary condition) is applied in this work. For example for the bottom wall, the unknown distribution functions are approximated by :

$\tilde{f}_{2}=\tilde{f}_{4}$

$\tilde{f}_{5}=r \tilde{f}_{7}+(1-r) \tilde{f}_{8}$

$\tilde{f}_{6}=r \tilde{f}_{8}+(1-r) \tilde{f}_{7}$

The accommodation factor, $r$,is chosen appropriately [15].

In analogy with the slip phenomenon, the temperature jump can be simulated on the microchannel walls by an equation as follows[16] :

$$
\Delta T_{w}=T_{\text {fluid }}(y \rightarrow \text { wall })-T_{w}=\left.\zeta \frac{\partial T_{\text {fluid }}}{\partial y}\right|_{w}
$$

where $\zeta$ is the temperature jump distance.For dimensionles temperature at the wall.It can be obtained from this eauation :

$$
\theta-\theta_{w}=\left.\frac{B}{\operatorname{Pr}} \frac{\partial \theta}{\partial y}\right|_{\mathrm{Y}=0,1}
$$

Using the diffuse scattering boundary condition, the temperature jump for the bottom wall is written as below in LBM, based on the internal energy distribution function [17] :

$g_{2,5,6}=\frac{3}{\rho_{w} e} g^{e}{ }_{2,5,6}\left(\rho_{w} u_{w} e_{w}\right)\left(g_{4}+g_{7}+g_{8}\right)$

The top wall temperature jump is also calculated similarly.

The local Nusselt number is given as [5]:

$$
N u_{x}=\left(\frac{k_{n f}}{k_{f}}\right) \frac{q_{y} D_{H}}{\Delta T k}=\left(\frac{k_{n f}}{k_{f}}\right) \frac{D_{H}(\partial T / \partial y)_{w}}{\left(T_{w}-T_{b u l k}\right)}
$$

\subsection{Grid independency and validation}

Trial computations have been performed using three mesh sizes, i.e. $700 \times 35,800 \times 40$ and $900 \times 45$ for nanoparticles concentration $\varphi=0$ and slip coefficient $B=0.01$. The 
numerical results for different grids are shown in Table 1, due to small difference between the results of the last two grid sizes, a uniform grid with $800 \times 40$ was chosen to obtain the best agreement between accuracy and computation time.

To validate the developed code, the comparison of the values obtained by Santra et al. [18] for the average Nusselt number (for different Reynolds number: $\operatorname{Re}=50, \mathrm{Re}=100$ and $\mathrm{Re}=200$ ) of a forced convection of cold $\mathrm{Cu}$-water nanofluid in a macro channel with hot walls. The figure 3 demonstrated good agreement with those of Santra et al. [18].

In the present computation, the Reynolds number and the Prandtl number are chosen to be $\operatorname{Re}=\rho_{n f} u_{n f} D_{H} / \mu_{n f}, \operatorname{Pr}=v_{n f} / \alpha_{n f}$ and are calculated for the nanofluid mixture at $\varphi=0 \%$ (pure water), $\varphi=0.02=2 \%$ and $\varphi=0.04=4 \%$, and the Reynolds number is fixed 1 . The effects of slip Velocity on the walls and heat transfer are examined, the slip Velocity coefficient is chosen from 0.005 to 0.02 .

Table 1

Thermophysical properties of $\mathrm{Ag}$ (Argent) as the nanoparticles and water as the base fluid (at average temperature from 27 to $34,7^{\circ} \mathrm{C}$ )

\begin{tabular}{ccccc}
\hline & $\begin{array}{c}\rho(\mathrm{Kg} / \\
\left.\mathrm{m}^{3}\right)\end{array}$ & $\begin{array}{c}\mathrm{K} \\
(\mathrm{W} / \mathrm{m} \\
\mathrm{K})\end{array}$ & $\begin{array}{c}\mu(\mathrm{Pa} . \mathrm{s} \\
)\end{array}$ & $\begin{array}{c}\mathrm{c}_{\mathrm{p}}(\mathrm{J} / \mathrm{K} \\
\mathrm{gK})\end{array}$ \\
\hline $\mathrm{Ag}$ & 3970 & 429 & - & 235 \\
Water & 997.1 & 0.613 & $\begin{array}{c}8.91 \times \\
10^{-4}\end{array}$ & 4179 \\
& & \multicolumn{3}{c}{} \\
\hline
\end{tabular}

Table 2

Grid Independency for $\operatorname{Re}=1, \operatorname{Pr}=0.7, \varphi=0$ and $\mathrm{B}=0.015$

\begin{tabular}{cccc}
\hline \multicolumn{5}{c}{ Grids } \\
\hline & $700 \times 35$ & $800 \times 40$ & $900 \times 45$ \\
\hline $\mathrm{Nu}$ & 7.18 & 7.23 & 7.25 \\
$C_{f} R e$ & 21.04 & 21.10 & 21.13
\end{tabular}

\section{Acknowledgments}

The first author is currently completing her doctorate. In addition, she did advanced internships at the Laboratory of physical Acoustics /Sapienza University to perform numerical simulations under the supervision of Prof. A. D'Orazio. The authors express their gratitude to the universities and institutes concerned for their assistance. 


\section{Results and discussions}

It is worth recalling that the laminar forced convection heat transfer of a $\mathrm{Ag} /$ water in a microchannel is studied numerically by using Lattice Boltzmann method.

Figures 4 and 5 portray the variations of $U_{s}$ and $\theta_{s}$ along the microchannel's walls at $\mathrm{B}=$ $0.005, \mathrm{~B}=0.01$ and $\mathrm{B}=0.02$ for $\varphi=0.04$. It can be depicted at the inlet, the slip velocity and temperature jump start from their maximum values and decrease asymptotically along the wall and approach constant values. Large values of B induce an improvement in both $U_{s}$ and $\theta_{s}$.

The effects of $\varphi$ on $N u_{x}$ and temperature jump ( $\left.\theta_{s}\right)$ along the microchannel wall are shown in Figures 6 and 7 at $\mathrm{B}=0.005$ for $\varphi=0, \varphi=0.02$ and $\varphi=0.04$, respectively. It is interesting to note that $N u_{x}$ and $\theta_{s}$ have the same tendency. Then, they start with largest value at entrance decrease to reach an asymptotic value along walls and approach constant values. Moreover, they increase with $\varphi$. However, this effect is more pronounced for $N u_{x}$. Figure 9 portrays that the temperature jump around the entrance region occurs a higher values, which has the most temperature gradient near the wall.

Figure 8 demonstrates the variations of $N u_{x}$ along the microchannel walls at $\mathrm{B}=0.005, \mathrm{~B}$ $=0.01$ and $\mathrm{B}=0.02$ for $\varphi=0, \varphi=0.02$ and $\varphi=0.04$. It can be noted $N u_{x}$ augments with $\varphi$; but declines with B. Temperature gradient between the nanofluid particles on the wall and their neighbor ones adjacent to the wall, diminishes at larger B; as a result $N u_{x}$ becomes less amount at recent case.

\section{Conclusion}

Laminar forced convection heat transfer of $\mathrm{Ag}-$ water nanofluid in a microchannel is simulated using a double population LBM-BGK method. The effects of different volume fractions of Argent nanoparticles and slip coefficient were investigated on the slip velocity, temperture jump and Nusselt number For $\mathrm{Re}=0,01$.

The numerical obtained results confirmed higher $\varphi$ corresponds to larger Nux. Moreover, it was stated that larger values of B induces a decreasing in Nux and larger values of $U_{s}$ and $\theta_{s}$. At the entrance region, the temperature jump reaches a high values along the microchannel walls especially which has the most temperature gradient between the walls and nanofluid. As a result, to increase Nux in micro liquid flows, it is recommended to use nanofluid with $\varphi=4 \%$ and at low values of slip coefficient as like $B=0.005$. However, the effect of $\varphi$ is more pronounced compared to B. 


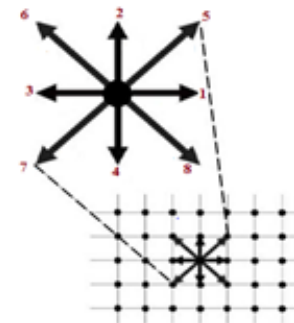

Fig. 1. Lattice structure for distribution function

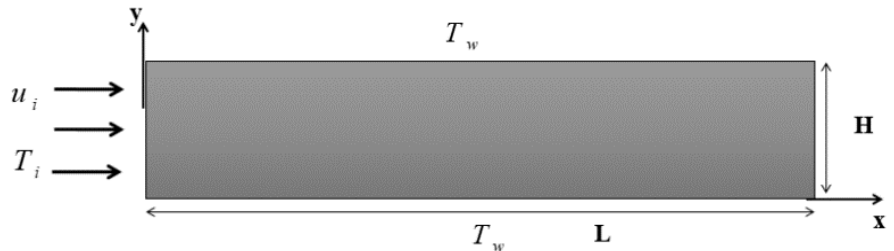

Fig.2.The shematic diagram of the microchannel

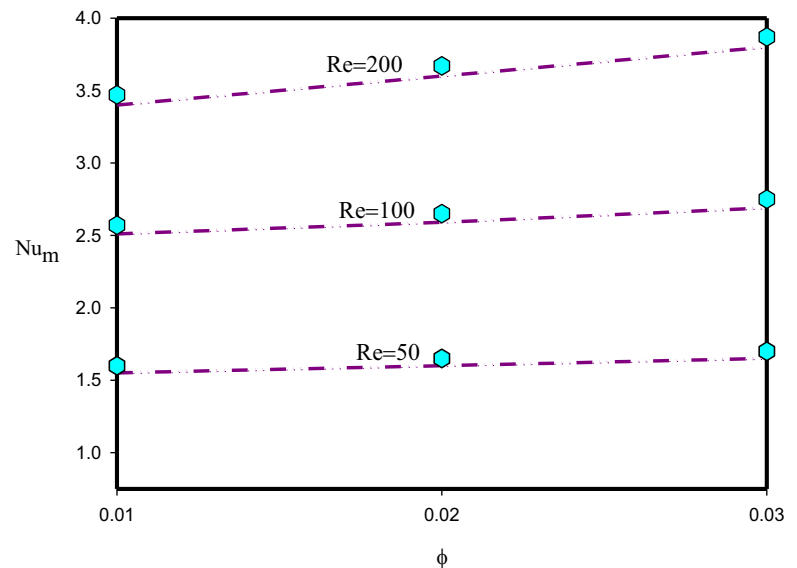

Fig. 3. Validation for the averaged Nusselt number vs. the results of Santra et al. [18](Line :present work, symbols : Santara et al.[18]) 


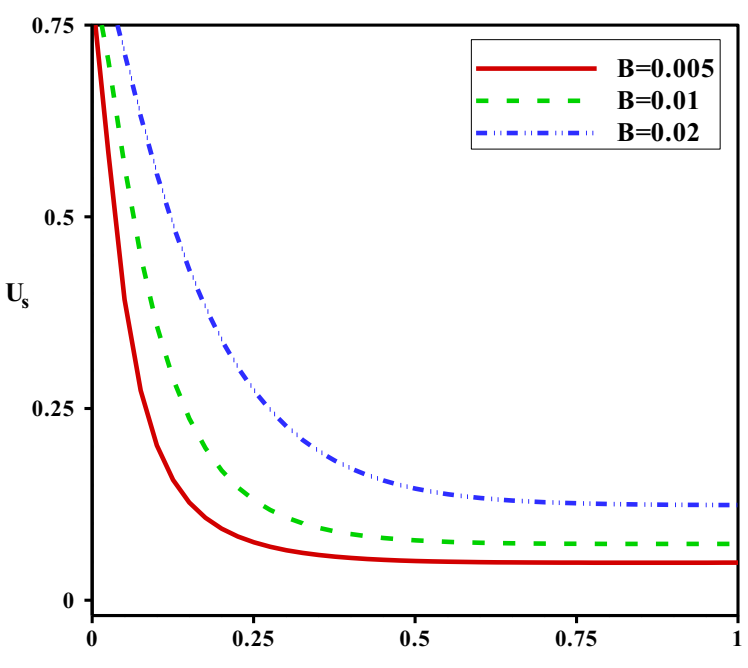

Fig.4. $U_{s}$ along the microchannel wall at $\mathrm{B}=0.005, \mathrm{~B}=0.01$ and $\mathrm{B}=0.02$ for $\phi=0.04$

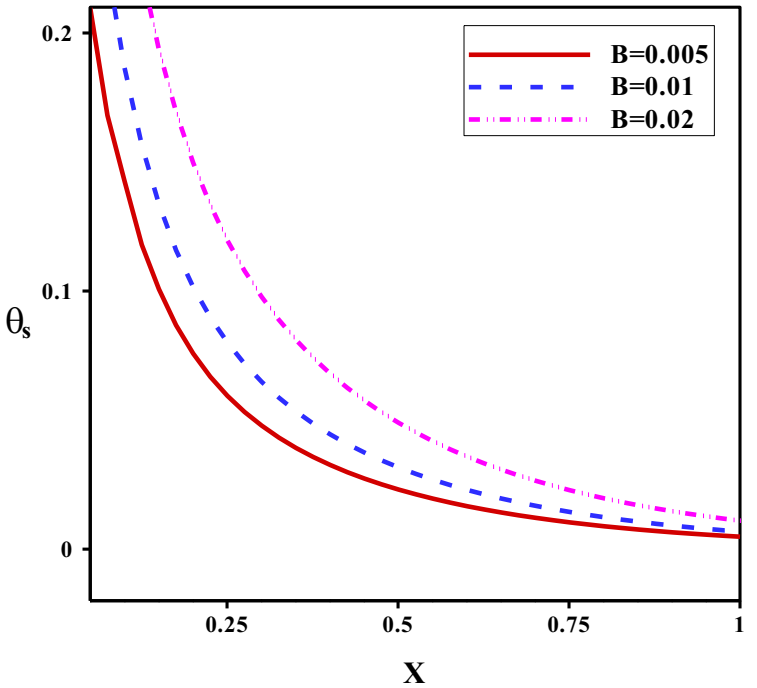

Fig.5. $\theta_{s}$ along the microchannel wall at $\mathrm{B}=0.005, \mathrm{~B}=0.01$ and $\mathrm{B}=0.02$ for $\phi=$ 0.04 


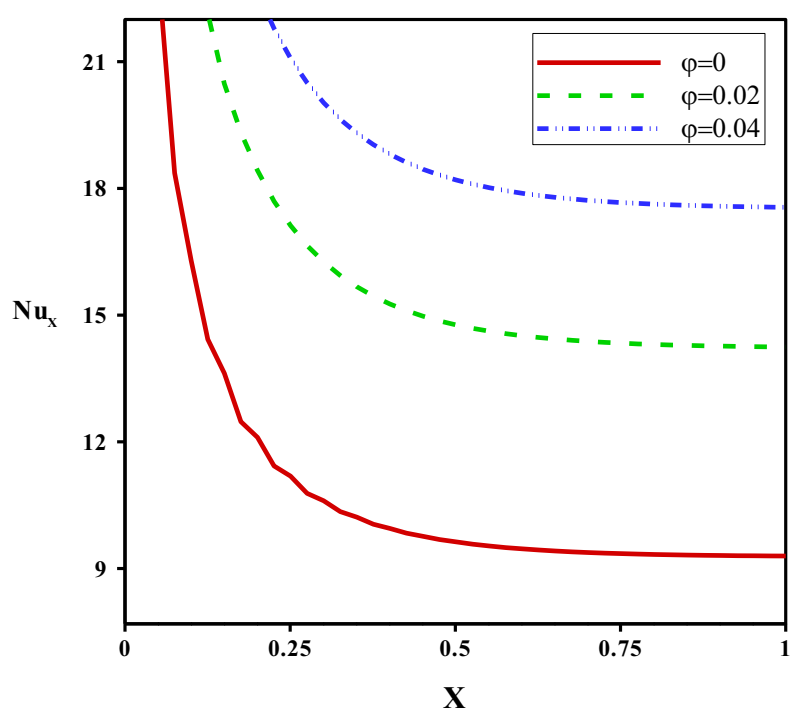

Fig.6. $N u_{x}$ along the microchannel wall at $\mathrm{B}=0.005$ for $\phi=0, \phi=0.02$ and $\phi=0.04$

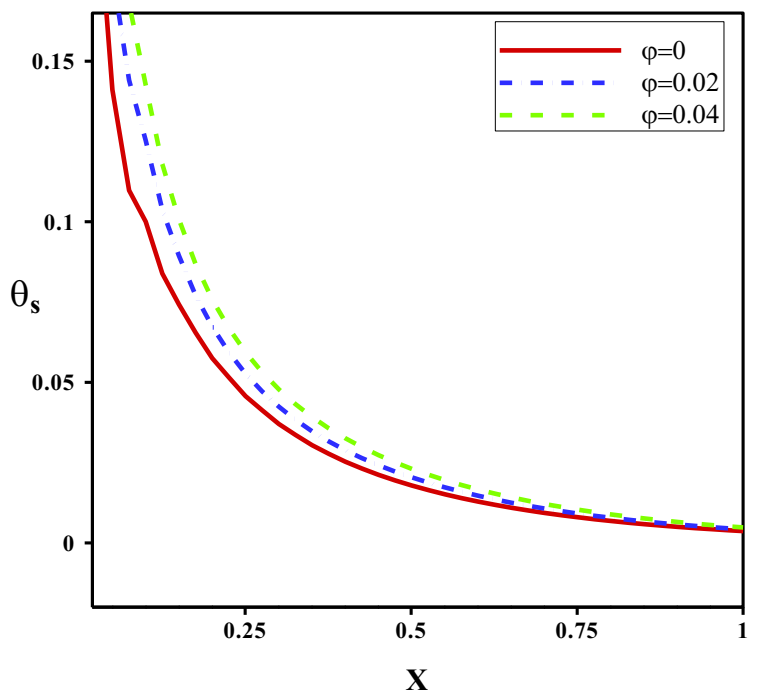

Fig.7. $\theta_{s}$ along the microchannel wall at $\mathrm{B}=0.005$ for $\phi=0, \phi=0.02$ and $\phi=$ 0.04 

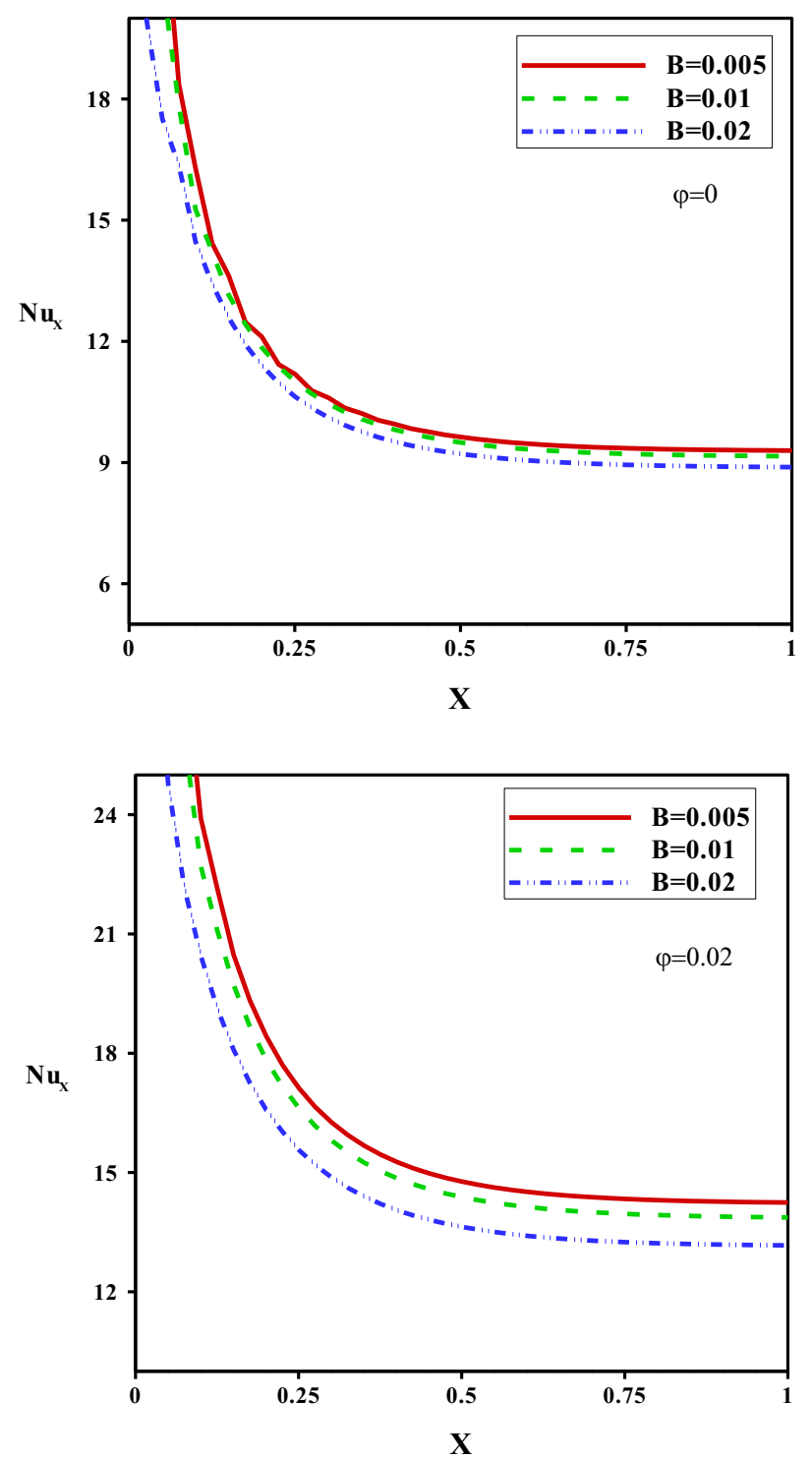


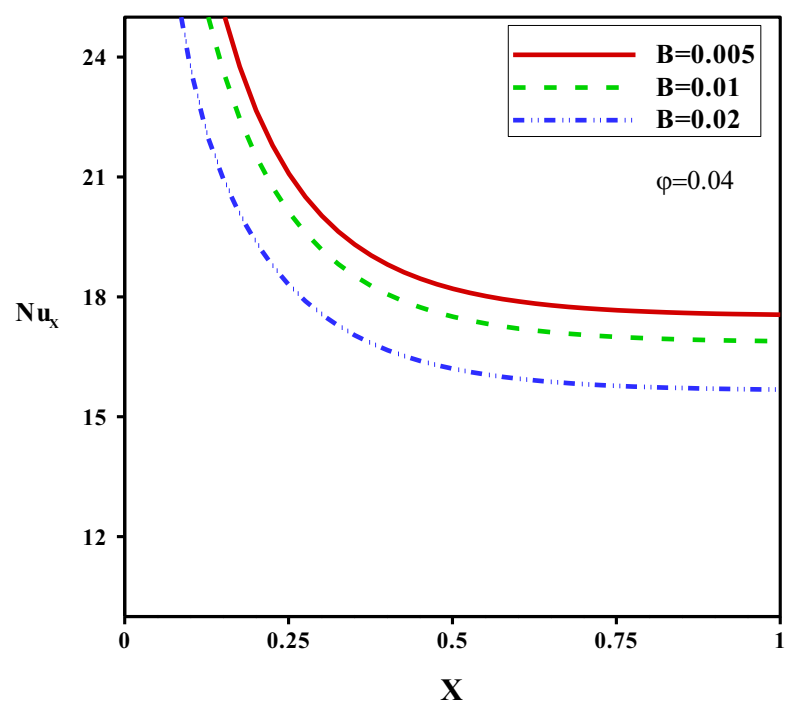

Fig.8.The variation of $N u_{x}$ along the microchannel wall at $\mathrm{B}=0.005, \mathrm{~B}=0.02$ and $\mathrm{B}=0.01$ for $\phi=0, \phi=0.02$ and $\phi=0.04$

\section{References}

1. S.M. Aminossadati, A. Raisi, B. Ghasemi, Effects of magnetic field on nanofluid forced convection in a partially heated microchannel, Int. J. Non-Linear Mech., 46, 1373-1382 (2011).

2. M. Kalteh, A. Abbassi, M. Saffar-Avval, J. Harting, Eulerian-Eulerian twophase numerical simulation of nanofluid laminar forced convection in a microchannel, Int. J. Heat Fluid Flow,32 ,107-116 (2011).

3. M. Mital, Semi-analytical investigation of electronics cooling using developing nanofluid flow in rectangular microchannels, Appl. Therm. Eng., 52, 321-327 (2013).

4. M. Mital, Analytical analysis of heat transfer and pumping power of laminar nanofluid developing flow in microchannels, Appl. Therm. Eng., 50, 429-436 (2013).

5. A. Karimipour,A H. Nezhad,A D’Orazio,M.H. Esfe,M. R. Safaei,E. Shirani, Simulation of copper-water nanofluid in a microchannel in slip flow regime using the lattice Boltzmann method,European Journal of mechanics B/Fluids , 49, 89-99 (2015).

6. C. Ay, C.W. Young, C.F. Young, Application of lattice Boltzmann method to the fluid analysis in a rectangular microchannel, Comput. Math. Appl., 64, 1065-1083(2012).

7. Y.T. Yang, F.H. Lai, Numerical study of flow and heat transfer characteristics of alumina-water nanofluids in a microchannel using the lattice Boltzmann method, Int. Commun. Heat Mass Transfer, 38,607-614 (2011).

8. ] Bird G 1994 Molecular gas dynamics and the direct simulation of gaz flows (Oxford).

9. Z. Guo, T.S. Zhao, Lattice Boltzmann model for incompressible flows through porous media, Physical Review E 66 ,036304/1-9 (2002). 
10. A.D’Orazio, S. Succi, Simulation two-dimensional thermal channel flows by means of a lattice Boltzmann method with new boundary conditions,Future Genet. Comput. Syst., 20,935-944 (2014).

11. H C Brinkman 1952 The viscosity of concentrated suspensions and solutions J. Chem. Phys. 20

12. A. D’Orazio, S. Succi, C.Arrighetti, Lattice Boltzmann simulation of open flows with heat transfer, Phys, Fluids , 15, 2778-2781(2003).

13. A. D’Orazio, M. Cercione, G.P. Celata, Application to naturel convection enclosed flows of a lattice Boltzmann BGK model coupled with a general purpose thermal boundary condition, Int. J. Therm. Sci., 43,575-586 (2004).

14. G D Ngoma and F Erchiqui F, Heat flux and slip effects on liquid flow in a microchannel INT. J. Therm. Sci., 46, 1076-1083 (2007).

15. G Karniadakis and A Beskok 2002 Micro flows: fundamentals and simulation (New York).

16. A. Akbarinia, M. Abdolzadeh, R. Laur, Critical investigation of heat transfer enhancement using nanofluids in microchannels with slip and non-slip flow regimes, Appl. Therm. Eng., 31, 556-565 (2001).

17. X.D. Niu, C. Shu, Y.T. Chew, A thermal lattice Boltzmann model with diffuse scattering boundary condition for micro thermal flows, Comp. Fluids., 36, 273-281 (2007).

18. A.K. Santara, S. Sen, N. Chakraborty, Study of heat transfer due to laminar flow of copper -water nanofluid through two isothermally heated parallel plates, Int. J. Therm. Sci., 48, 391-400 (2009). 